\title{
Diseño y generación de transmisiones de potencia por correa trapecial en Solidworks mediante una aplicación en Visual Basic
}

\author{
Power transmission V-Belt's design and generation in SolidWorks \\ by a Visual Basic application
}

Fecha de Recepción: 07 de octubre de 2013

Fecha de Aprobación: 17 de noviembre de 2013

\author{
Alejandro Ozaeta Eidelman* \\ Luis Carlos Flórez García* \\ Oscar Fabián Higuera Cobos ${ }^{* *+*}$
}

\section{Resumen}

Se presenta el procedimiento de diseño de transmisiones de potencia por correa trapecial según la norma BS 3790, mediante una aplicación desarrollada en Microsoft Visual Studio 2008 y utilizando el lenguaje de programación Visual Basic. El programa utiliza los parámetros de operación de la transmisión ingresada por el usuario, y mediante los cálculos estandarizados y una base de datos conformada por diversos catálogos de poleas y correas, muestra todas las transmisiones que satisfacen los requerimientos ingresados; además, permite la generación del modelo sólido de cada transmisión en SolidWorks.

Palabras clave: Correa trapecial, Transmisiones de potencia, SolidWorks, Visual Basic.

\begin{abstract}
This article presents the design procedure for V-belt's power transmission, according to standard BS 3790 , by using an application developed in the Microsoft Visual Studio 2008 and applying the Visual Basic programming language. The program utilizes the transmission input operating parameters provided by the user, and by the standard calculations and a database made up of several catalogs of pulleys and belts, it shows all transmissions admitted which meet the requirements, it also allows a solid model generation of each transmission in SolidWorks.
\end{abstract}

Keywords: V-belt, Power Transmission, SolidWorks, Visual Basic.

\footnotetext{
* Universidad Tecnológica de Pereira (Colombia). alejandroozaeta@gmail.com

** M.Sc. Universidad Tecnológica de Pereira (Colombia). luiscflorez@gmail.com

***Ph.D. Universidad Tecnológica de Pereira (Colombia). osfahico@gmail.com
} 


\section{INTRODUCCIÓN}

Gracias a los avances tecnológicos y a las mejoras en los métodos de producción y en la calidad de los materiales de las correas de transmisión, en la actualidad se ha extendido el uso de las transmisiones por correa trapecial. De allí la importancia de agilizar el diseño de este tipo de transmisiones para reducir el tiempo y el costo de su fabricación, para ayudar a estudiantes, ingenieros y diseñadores a seleccionar el diseño apropiado de acuerdo con los requerimientos establecidos y para hacer más eficiente el cálculo de transmisiones, que podría llegar a ser un proceso arduo y repetitivo.

El factor más importante que determina la capacidad de tracción en una transmisión por correa es su tensión inicial; por ello, es importante calcular debidamente el valor de esta tensión, y controlarlo frecuentemente durante el uso de la transmisión, especialmente en accionamientos modernos, donde se exige mayor capacidad de carga para cada correa [1].

V-Belts Design View es una aplicación de diseño y generación de transmisiones por correa trapecial que automatiza los cálculos de diseño según la capacidad de tracción, y determina la tensión estática inicial y su durabilidad aproximada, con base en los procedimientos establecidos por algunos fabricantes (GoodYear y Gates), en la norma BS 3790 e ISO 5292. Adicionalmente, genera el modelo sólido de la transmisión, con el fin de facilitar su visualización y reducir el tiempo de su generación gráfica .

\section{Metodología PARA diSEÑAR TRANSMISIONES POR CORREA TRAPECIAL}

Para diseñar una transmisión por correa trapecial se deben conocer ciertas condiciones de trabajo, tales como [2]:
- Característica de la máquina motriz y accionada

- Potencia por transmitir $(\mathrm{N})$

- Frecuencia de rotación de una de las dos poleas

- Relación de transmisión (i) o frecuencia de rotación de la otra polea

\section{A. Selección del factor de servicio $(f)$}

Es un factor que prevé los incrementos de carga que se producen en las correas, de acuerdo con los diferentes tipos de máquinas en las que se instalará la transmisión, con la cantidad de horas diarias de trabajo y con la relación de transmisión que se desea (ver Tabla 1).

\section{B. Cálculo de la potencia de diseño $\left(N_{D}\right)$}

Este valor se define como la potencia máxima por transmitir, teniendo en cuenta las condiciones consideradas por el factor de servicio [3], como se aprecia en (1).

$$
\mathrm{N}_{\mathrm{D}}=\mathrm{N} * \mathrm{f}_{\mathrm{s}}[\mathrm{kW}](1)
$$

\section{Selección del perfil de la correa}

De acuerdo con la velocidad de la polea más rápida y la potencia de diseño, es posible seleccionar el tipo de perfil de la correa por implementar en la transmisión, mediante el nomograma de la Fig. 1.

Si el punto de corte entre la potencia y la velocidad se encuentra cerca de una de las líneas que dividen las regiones de cada correa, se sugiere realizar el diseño con ambos perfiles y adoptar la transmisión más económica [1]. 
TABLA 1

FACTOR DE SERVICIO (FS) [3]

\begin{tabular}{|c|c|c|c|c|c|c|c|}
\hline \multicolumn{2}{|r|}{ Máquina accionada } & \multicolumn{6}{|c|}{ Máquina motriz } \\
\hline \multirow[t]{3}{*}{ Clase } & \multirow[t]{3}{*}{ Ejemplos } & \multirow{2}{*}{\multicolumn{3}{|c|}{$\begin{array}{l}\text { Motores eléctricos (C.A. de } \\
\text { arranque suave, C.D. con } \\
\text { arrancador estrella delta, } \\
\text { C.D. Shunt), motores de } \\
\text { combustión interna con más } \\
\text { de } 4 \text { cilindros, máquinas con } \\
\text { acoples flexibles. } \\
\text { Horas de trabajo diarias }\end{array}$}} & \multicolumn{3}{|c|}{$\begin{array}{l}\text { Motores eléctricos (C.A. de } \\
\text { arranque directo, C.D. serie } \\
\text { y compuesto), máquinas } \\
\text { de combustión interna con } \\
\text { menos de } 4 \text { cilindros. }\end{array}$} \\
\hline & & & & & Hor & de trabaj & iarias \\
\hline & & $<10$ & 10 a 16 & $>16$ & $<10$ & 10 a 16 & $>16$ \\
\hline $\begin{array}{c}1 \\
\text { (servicio } \\
\text { ligero) }\end{array}$ & $\begin{array}{l}\text { Agitador (densidad uniforme). } \\
\text { Ventilador y soplador ( }<7.5 \mathrm{~kW}) \text {. } \\
\text { Compresor y bomba centrífuga. } \\
\text { Banda transportadora (carga } \\
\text { uniforme). } \\
\end{array}$ & 1.0 & 1.1 & 1.2 & 1.1 & 1.2 & 1.3 \\
\hline $\begin{array}{c}2 \\
\text { (servicio } \\
\text { medio) }\end{array}$ & $\begin{array}{c}\text { Agitador (densidad variable). } \\
\text { Ventilador y soplador }(>7.5 \mathrm{~kW}) \text {. } \\
\text { Compresor y bomba rotatoria. Banda } \\
\text { transportadora (carga no uniforme), } \\
\text { Generador, Lavadora, Eje de } \\
\text { transmisión. } \\
\text { Máquina herramienta, Imprenta, } \\
\text { Máquina para madera. }\end{array}$ & 1.1 & 1.2 & 1.3 & 1.2 & 1.3 & 1.4 \\
\hline $\begin{array}{c}3 \\
\text { (servicio } \\
\text { pesado) }\end{array}$ & $\begin{array}{c}\text { Máquina para fabricar ladrillo. } \\
\text { Elevador, Compresor y Bomba } \\
\text { reciprocante. Transportadora (carga } \\
\text { pesada), Montacargas, Molino de } \\
\text { percusión, Pulverizadora, Prensa, } \\
\text { Cizalla, Maquinaria para caucho, } \\
\text { Vibrador, Maquinaria textil } \\
\end{array}$ & 1.2 & 1.3 & 1.4 & 1.4 & 1.5 & 1.6 \\
\hline $\begin{array}{c}4 \\
\text { (servicio extra } \\
\text { pesado) }\end{array}$ & $\begin{array}{c}\text { Troqueladora, Laminadora } \\
\begin{array}{c}\text { Trituradora circular, de mordazas, de } \\
\text { rodillos }\end{array} \\
\text { Molino triturador de bolas y de barras }\end{array}$ & 1.3 & 1.4 & 1.5 & 1.5 & 1.6 & 1.8 \\
\hline & $\begin{array}{r}\text { Nota: Para transmisiones } \\
\operatorname{Si}(1.0<\mathrm{i}<1.24) \mathrm{n} \\
\mathrm{Si}(1.25<\mathrm{i}<1.74) \\
\mathrm{Si}(1.75<\mathrm{i}<2.49) \\
\mathrm{Si}(2.5<\mathrm{i}<3.49) \mathrm{n} \\
\mathrm{Si}(\mathrm{i}>3.5) \text { mult }\end{array}$ & $\begin{array}{l}\text { con red } \\
\text { ultiplic } \\
\text { nultipli } \\
\text { nultipli } \\
\text { ultiplic } \\
\text { iplique }\end{array}$ & $\begin{array}{l}\text { ción de ve } \\
\text { fs por } 1.0 \\
\text { fs por } 1.0 \\
\text { fs por } 1.1 \\
\text { fs por } 1.1 \\
\text { oor } 1.25\end{array}$ & & & & \\
\hline
\end{tabular}




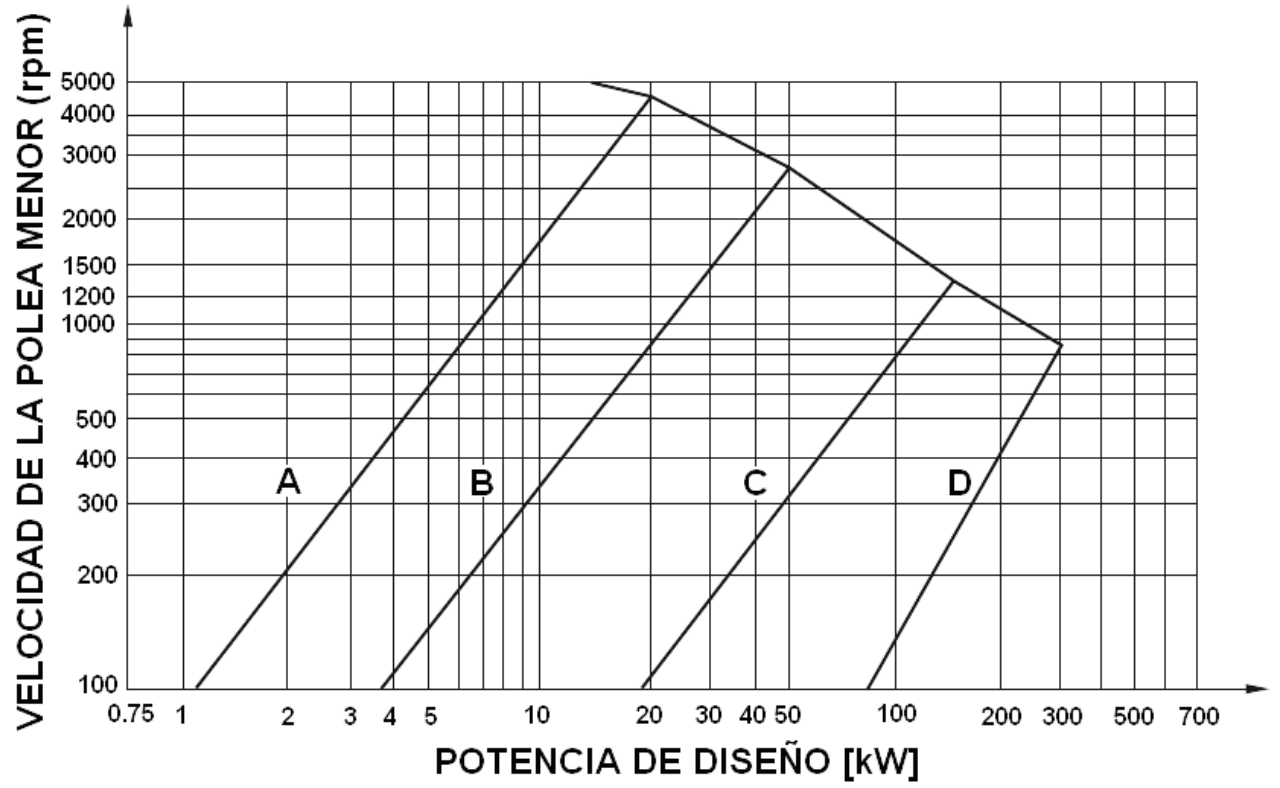

FIG. 1. Recomendaciones para seleccionar el perfil en correas clásicas [3]

\section{Selección del diámetro de las poleas}

Para este proceso es necesario tener en cuenta que se deben escoger las poleas con un diámetro mayor al mínimo recomendado para cada perfil (ver Tabla 2), y cumplir con la relación de transmisión (i) deseada (2).

$$
\mathrm{i}=\mathrm{d}_{2} / \mathrm{d}_{1}(2)
$$

Siendo:

- $\quad \mathrm{d}_{1}$ : Diámetro de la polea motriz

- $\quad \mathrm{d}_{2}$ : Diámetro de la polea accionada

La selección de las poleas se realiza con base en los catálogos que proporcionan los fabricantes de transmisiones por correa trapecial.

\section{Tabla 2}

DIÁMETRO MÍNIMO RECOMENDADO PARA LA POLEA MENOR EN FUNCIÓN DE SU PERFIL [3]

\begin{tabular}{|c|c|c|c|c|}
\hline Perfil & A & B & C & D \\
\hline $\begin{array}{c}\text { Diámetro mínimo } \\
{[\mathrm{mm}]}\end{array}$ & 71 & 112 & 180 & 355 \\
\hline
\end{tabular}

\section{E. Cálculo de la velocidad de la correa}

Este procedimiento se realiza para comprobar que las correas no superen la velocidad límite permisible para el perfil utilizado $(30 \mathrm{~m} / \mathrm{s}$ para correas en $\mathrm{V}$ clásicas) [3]; sin embargo, se recomienda que la velocidad se encuentre cerca a este valor para poder transmitir la potencia requerida con el mínimo número de correas [4].

La velocidad de las correas está determinada por la siguiente fórmula [2]:

$$
\mathrm{v}=\frac{\mathrm{n}_{1} * \pi * \mathrm{~d}_{1}}{60000}=\frac{\mathrm{n}_{2} * \pi * \mathrm{~d}_{2}}{60000}\left[\frac{m}{s}\right](3)
$$

Siendo:

- $\quad \mathrm{n}_{1}$ y $\mathrm{n}_{2}:$ Frecuencia de rotación de las poleas [rpm]

- $\quad \mathrm{d}_{1} \mathrm{y} \mathrm{d}_{2}$ : Diámetro de las poleas $[\mathrm{mm}]$

F. Cálculo de la longitud de la correa $\left(L_{n}\right)$ y de la distancia entre los centros de la poleas (a)

Si las características iniciales de la transmisión no sugieren una distancia entre centros aproximada 
$\left(a_{0}\right)$, se recomienda utilizar la siguiente fórmula para calcular un valor inicial de la distancia entre centros (Norma DIN 7753) [2]:

$$
0.7 *\left(d_{1}+d_{2}\right)<a_{0}<2 *\left(d_{1}+d_{2}\right)(4)
$$

Luego de estimar la distancia entre centros, se calcula la longitud aproximada de la correa $\left(\mathrm{L}_{0}\right)$, mediante la siguiente expresión [3]:
$\mathrm{L}_{0}=2 * \mathrm{a}_{0}+\frac{\pi}{2} *\left(\mathrm{~d}_{2}+\mathrm{d}_{1}\right)+\frac{\left(\mathrm{d}_{2}-\mathrm{d}_{1}\right)^{2}}{4 * \mathrm{a}_{0}}(5)$

Con base en el cálculo de la longitud aproximada $\left(\mathrm{L}_{0}\right)$ y en los catálogos que brindan los fabricantes de correas trapeciales, se selecciona una correa con longitud primitiva $\left(\mathrm{L}_{\mathrm{n}}\right)$ cercana a la aproximada. Posteriormente, se recalcula la distancia entre centros, como se muestra a continuación [3].

$$
\mathrm{a}=\frac{\mathrm{L}_{\mathrm{n}}}{4}-\pi * \frac{\left(\mathrm{d}_{1}+\mathrm{d}_{2}\right)}{8}+\sqrt{\left(\frac{\mathrm{L}_{\mathrm{n}}}{4}-\pi * \frac{\left(\mathrm{d}_{1}+\mathrm{d}_{2}\right)}{8}\right)^{2}-\frac{\left(\mathrm{d}_{2}-\mathrm{d}_{1}\right)^{2}}{8}}(6)
$$

Las expresiones mostradas solamente son válidas para transmisiones abiertas con dos poleas. Si la transmisión por diseñar tiene características diferentes a las mencionadas, se recomienda realizar un dibujo a escala de la transmisión para determinar las distancias entre los centros de las poleas; es importante mencionar que este factor también depende del espacio de trabajo.

\section{G. Verificación de los ciclos de flexión por segundo (i)}

Pruebas experimentales han demostrado que la flexión de las correas durante el contacto con cada polea influye de manera negativa en la vida útil de la transmisión, debido al calentamiento excesivo de las correas en dichos puntos [4]; es por este motivo que se recomienda no sobrepasar el valor límite de flexiones por segundo durante el funcionamiento normal de una transmisión por correa trapecial $\left(40 \mathrm{~s}^{-1}\right)$ [2].

La cantidad de flexiones por segundo se calcula mediante la siguiente expresión [2]:

$$
\mathrm{i}_{\mathrm{f}}=1000 * \mathrm{n}_{\mathrm{p}} * \frac{\mathrm{v}}{\mathrm{L}_{\mathrm{n}}}\left[\mathrm{s}^{-1}\right](7)
$$

Siendo:

- $\quad n_{p}$ : Número de poleas en la transmisión

- v: Velocidad de la correa $[\mathrm{m} / \mathrm{s}]$

\section{- $\mathrm{L}_{\mathrm{n}}$ : Longitud primitiva de la correa $[\mathrm{mm}]$}

\section{H. Verificación del ángulo de contacto ( $\alpha)$}

Para transmisiones por correa trapecial es indispensable conocer el ángulo de contacto entre la correa y la polea de menor diámetro, debido a la influencia que esta característica tiene en relación con la capacidad de tracción. Se recomienda que este valor sea menor a $120^{\circ}$, para no causar un rápido deterioro de las correas [2].

Para calcular el ángulo de contacto puede ser empleada la fórmula que se muestra a continuación [2]:

$$
\alpha=2 * \cos ^{-1}\left(\frac{\mathrm{d}_{2}-\mathrm{d}_{1}}{2 * \mathrm{a}}\right)\left[^{\circ}\right]_{(8)}
$$

Siendo:

- $\quad \mathrm{d}_{1}$ : Diámetro primitivo de la polea menor [mm]

- $\quad \mathrm{d}_{2}$ : Diámetro primitivo de la polea mayor [mm]

- a: Distancia entre los centros [mm]

Para transmisiones con más de dos poleas es recomendable realizar el dibujo esquemático a escala para determinar los ángulos de contacto. 
I. Cálculo de la potencia nominal transmisible por correa $\left(N_{1}\right)$

Aunque no existe una exacta coincidencia de las magnitudes de potencia nominal transmisible por correa entre los diferentes fabricantes y normas, en la actualidad ha tenido gran aceptación por muchos fabricantes de transmisiones por correa la siguiente fórmula para el cálculo de la potencia, brindada por la RMA (Rubber Manufacturers Association) [5]:

$$
N_{1}=d \cdot r \cdot\left[K_{1}-\frac{K_{2}}{d}-K_{3} \cdot(d \cdot r)^{2}-K_{4} \cdot \log (d \cdot r)\right]+K_{2} \cdot r \cdot\left(1-\frac{1}{K_{i}}\right)(9)
$$

Siendo:

- $\quad \mathrm{N}_{1}$ : Potencia transmisible por correa $[\mathrm{kW}]$

- d: Diámetro primitivo de la polea menor [mm]

- r: Frecuencia de rotación de la polea más rápida $[\mathrm{rpm}]$ dividida por mil

- $\quad \mathrm{K}_{\mathrm{i}}$ : Factor por razón de transmisión

- $\mathrm{K}_{1}, \mathrm{~K}_{2}, \mathrm{~K}_{3}$ y $\mathrm{K}_{4}$ : Factores empíricos calculados por los fabricantes de transmisiones por correa trapecial

El factor $\mathrm{K}_{\mathrm{i}}$ se determina con la siguiente expresión y se resumen en la Tabla 3 [5]:

$$
\mathrm{K}_{\mathrm{i}}=\frac{1}{1+0.35 \cdot \log \left[\frac{1+10\left\{-\left(\frac{1}{0.35}\right)\left(1-\frac{1}{\mathrm{i}}\right)\right\}}{2}\right]}(10)
$$

Donde i es la razón de transmisión $\left(\mathrm{n}_{1} / \mathrm{n}_{2}\right)$.

\section{TABla 3}

FACTORES EMPÍRICOS $\left(\mathrm{K}_{1}, \mathrm{~K}_{2}, \mathrm{~K}_{3}\right.$ Y $\left.\mathrm{K}_{4}\right)$ SEGÚN EL PERFIL DE LA CORREA [5]

\begin{tabular}{|c|c|c|c|c|}
\hline Perfil & $\mathrm{K}_{1}$ & $\mathrm{~K}_{2}$ & $\mathrm{~K}_{3}$ & $\mathrm{~K}_{4}$ \\
\hline A & 0,041 & 1,954 & $1,32 * 10^{-8}$ & 0,0036 \\
\hline B & 0,065 & 4,361 & $2,18 * 10^{-8}$ & 0,0056 \\
\hline C & 0,108 & 10,22 & $3,70 * 10^{-8}$ & 0,010 \\
\hline D & 0,199 & 29,35 & $7,10 * 10^{-8}$ & 0,017 \\
\hline
\end{tabular}

\section{J. Cálculo de la cantidad de correas (z)}

La cantidad de correas en una transmisión se determina fundamentalmente de acuerdo con la potencia por transmitir y la potencia nominal transmisible por correa [2]:

$$
\mathrm{z}=\frac{\mathrm{N} * \mathrm{f}_{\mathrm{s}}}{\mathrm{N}_{1} * \mathrm{C}_{\alpha} * \mathrm{C}_{\mathrm{L}}}(11)
$$

Siendo:

- z: Número de correas de la transmisión

- $\quad$ N: Potencia por transmitir $[\mathrm{kW}]$

- $\mathrm{f}_{\mathrm{s}}$ : Factor de servicio

- $\mathrm{N}_{1}$ : Potencia nominal transmisible por correa $[\mathrm{kW}]$

- $\mathrm{C}_{\alpha}$ : Coeficiente de ángulo de contacto

- $\mathrm{C}_{\mathrm{L}}$ : Coeficiente por corrección de la longitud

Donde:

- $\mathrm{C}_{\alpha}=1.25 *\left(1-5^{\frac{-\alpha}{180}}\right)$

- $\alpha$ : Ángulo de contacto de la polea menor en grados [6]

- $\quad C_{L}=1+\left[\frac{\log \left(L_{n}\right)-\log \left(L_{b}\right)}{\log \left(L_{b}\right)-1.65}\right](13)$

- $\mathrm{L}_{\mathrm{n}}$ : Longitud primitiva de la correa $[\mathrm{mm}]$

- $\mathrm{L}_{\mathrm{b}}$ : Longitud base estándar [mm] [5]

Los valores de la longitud base se resumen en la Tabla 4. 
TABla 4

LONGITUD BASE ESTÁNDAR POR PERFIL (LB) [3]

\begin{tabular}{|l|c|c|c|c|}
\hline Perfil & A & B & C & D \\
\hline $\mathrm{L}_{\mathrm{b}}[\mathrm{mm}]$ & 1730 & 2280 & 3800 & 6375 \\
\hline
\end{tabular}

III. Proceso de diseño mediante la aplicación en Visual Basic "V-Belts DESIGN VIEW"

Las funciones de la aplicación creada están basadas en la utilización de cuatro módulos, desarrollados para facilitar el manejo del software y enmarcar cada utilidad en función de las necesidades del usuario.

\section{A. Módulo de dibujo personalizado}

Permite generar el modelo sólido de la transmisión de acuerdo con las dimensiones ingresadas por el usuario (Fig. 2).

Para impedir una generación defectuosa de la transmisión, se desarrollaron funciones que detectan dimensiones inconsistentes y muestran mensajes de error, informando al usuario qué dato fue ingresado de manera incorrecta, y algunas veces sugiriendo el rango de longitud admisible para estas dimensiones.

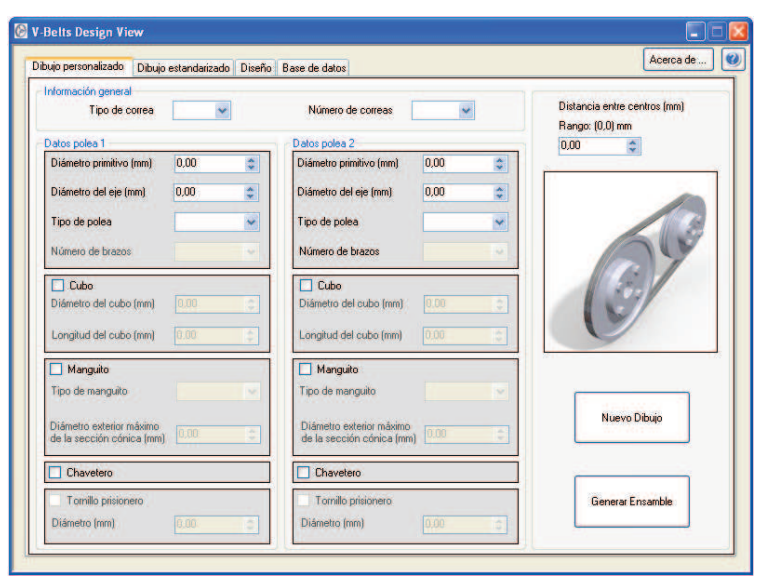

Fig. 2. Módulo de dibujo personalizado

\section{B. Módulo de dibujo estandarizado}

Permite generar el modelo sólido de la transmisión con base en la selección de las poleas y correas existentes en la base de datos, las cuales fueron ingresadas de acuerdo con las dimensiones estipuladas por algunas firmas productoras de transmisiones por correa en V (Fig. 3). Los datos utilizados para crear dicha base fueron tomados de cuatro catálogos proporcionados por fabricantes de transmisiones por correa trapecial:

- Optibelt (empresa alemana)

- $\quad$ SIT Spa (empresa italiana)

- SIT (empresa española)

- NBC Group Ltd (empresa del Reino Unido)

- Rexon (empresa colombiana)

Con el fin de enmarcar cada proceso para la selección de las transmisiones se establecieron cinco paneles:

Panel de Búsqueda: enél se ingresan y seleccionan las características de cada polea para su búsqueda automática.

Panel de Poleas: aquí se muestran todas las poleas con las características deseadas para su posterior selección.

Panel de Correas: en este se visualizan todas las correas con las características deseadas y admisibles para su posterior selección.

Panel para el ingreso del diámetro de cada eje: este presenta la opción de ingresar o seleccionar el diámetro de cada eje de acuerdo con el tipo de sujeción utilizado por cada polea (con o sin manguito).

Panel de Elementos seleccionados: en este se muestran cada uno de los elementos escogidos, y permite eliminar las selecciones realizadas. 


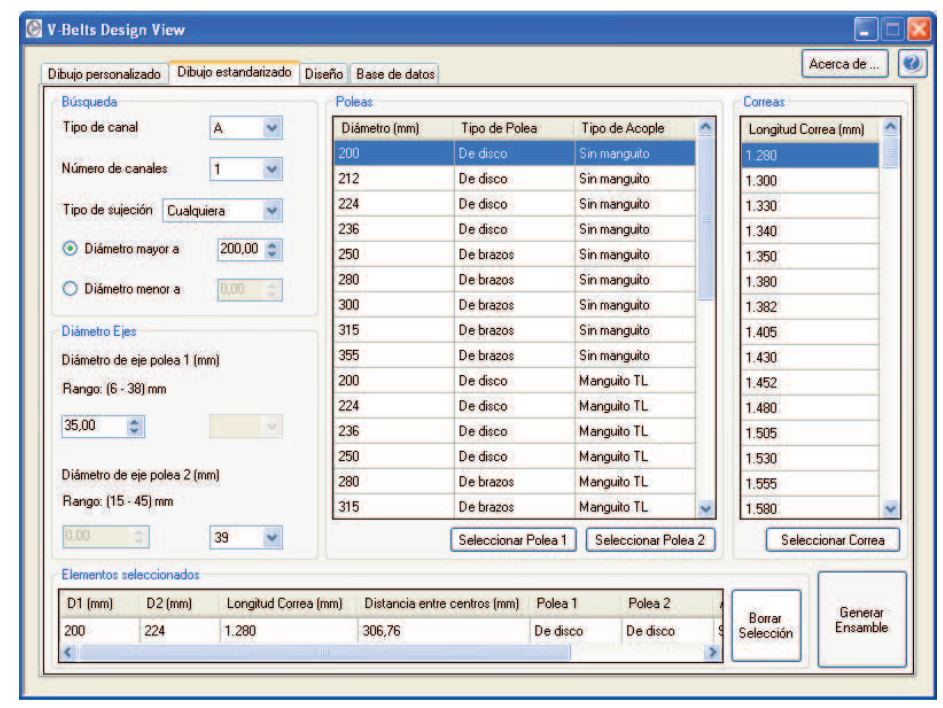

Fig. 3. Módulo de dibujo estandarizado

\section{Módulo de diseño}

Permite realizar el diseño de transmisiones por correa en $\mathrm{V}$ de acuerdo con los requerimientos de operación establecidos por el usuario; además, establece la opción de generar el modelo sólido de cada una de las transmisiones arrojadas por la aplicación (Fig. 4).

Durante el proceso para definir los pasos para el cálculo de transmisiones por correa se precisaron las variables de entrada y salida requeridas para tal fin, siendo estas:

\section{Variables de entrada:}

- Potencia por transmitir en kilovatios

- Velocidad de giro de la máquina motriz en revoluciones por minuto

- Tipo de máquina motriz

- Horas de trabajo diarias de la transmisión

- Tipo de máquina accionada

- Velocidad de giro de la máquina accionada en revoluciones por minuto o la relación de transmisión deseada

- Tolerancias de la relación de transmisión.
Estas variables fueron definidas para permitir que el programa pueda calcular transmisiones dentro de un rango cercano a la relación de transmisión deseada.

- Distancia entre ejes, máxima y mínima (opcional)

- Diámetro de los ejes (opcional)

- Tipo de acople

- Constantes de diseño. Algunos fabricantes brindan en sus catálogos las constantes para el cálculo de la potencia útil transmisible por correa, las cuales presentan valores variables de acuerdo con el tipo de fabricación y los materiales de las correas; por este motivo es necesario solicitarle al usuario seleccionar las constantes por utilizar para el desarrollo del respectivo proceso.

Variables de salida:

- Perfil de la correa

- Número de correas

- Diámetros de las poleas

- Longitud de las correas

- Distancia entre centros 
- Relación de transmisión teórica y real

- Velocidad teórica y real de la máquina accionada

- Tipos de polea

- Tipos de acople

- Diámetro de los ejes

- Fuerza en el ramal tenso y flojo por correa
- Tensión estática recomendada

- Vida útil aproximada de las correas

En este módulo se desarrollaron algunas funciones para evitar que el usuario ingrese valores de velocidad, potencia y relación de transmisión superiores al rango manejado en las transmisiones por correa.

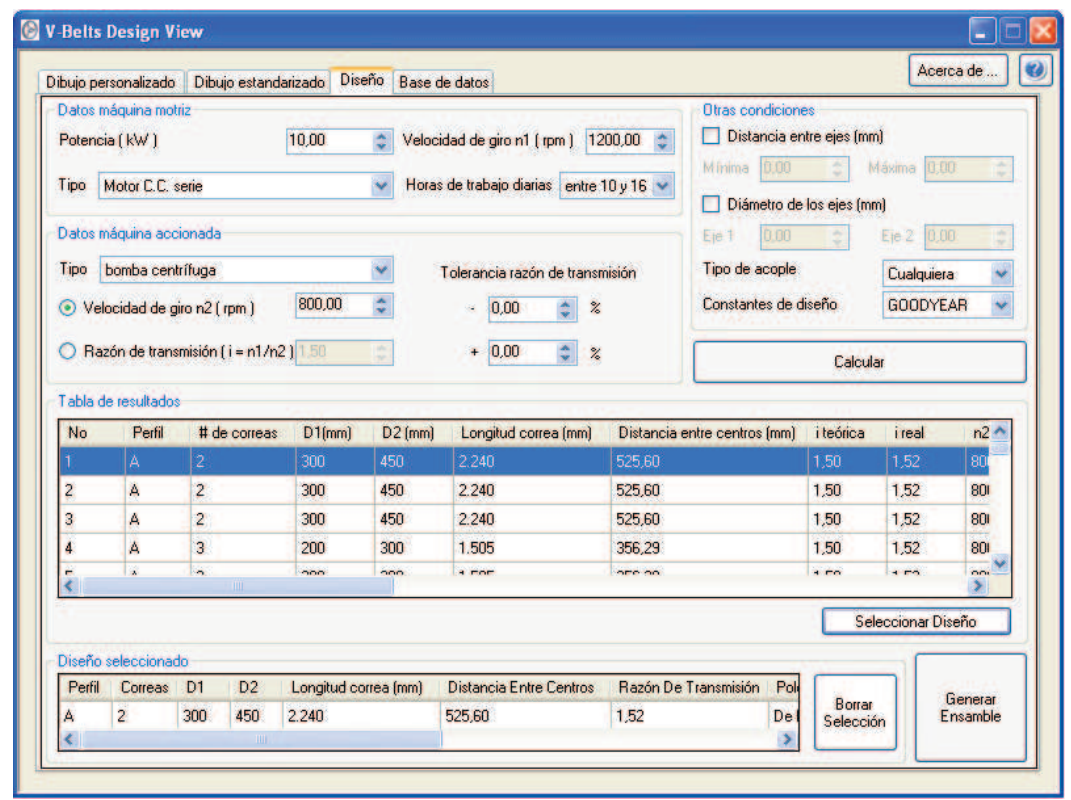

Fig. 4. Módulo de diseño

D. Módulo de ingreso de elementos a la base de datos

Permite el ingreso de nuevas poleas y correas a la base de datos para su posterior dibujo o utilización en los cálculos de diseño, teniendo en cuenta que las dimensiones ingresadas deben encontrarse dentro de los rangos normales establecidos por los fabricantes de transmisiones y las normas de estandarización utilizadas (Fig. 5).

Este módulo fue desarrollado pensando en el crecimiento del mercado y en la fabricación de nuevos productos: de esta manera es posible ampliar la base de datos con la cual se realizan las transmisiones en el módulo de dibujo estandarizado y se efectúan los cálculos en el módulo de diseño.

\section{E. Generación del modelo sólido de la transmisión}

Para poder generar un ensamble correctamente, todas las piezas que lo componen deben haber sido creadas y debidamente guardadas, función que antes se realizaba en una única dirección, impuesta por la aplicación; sin embargo, para brindar un mejor manejo del programa y permitir guardar el dibujo de la transmisión en cualquier ruta deseada por el usuario, se creó el cuadro de diálogo "Guardar Ensamble", el cual aparece justo después de oprimir el botón generar ensamble (Fig. 6). 


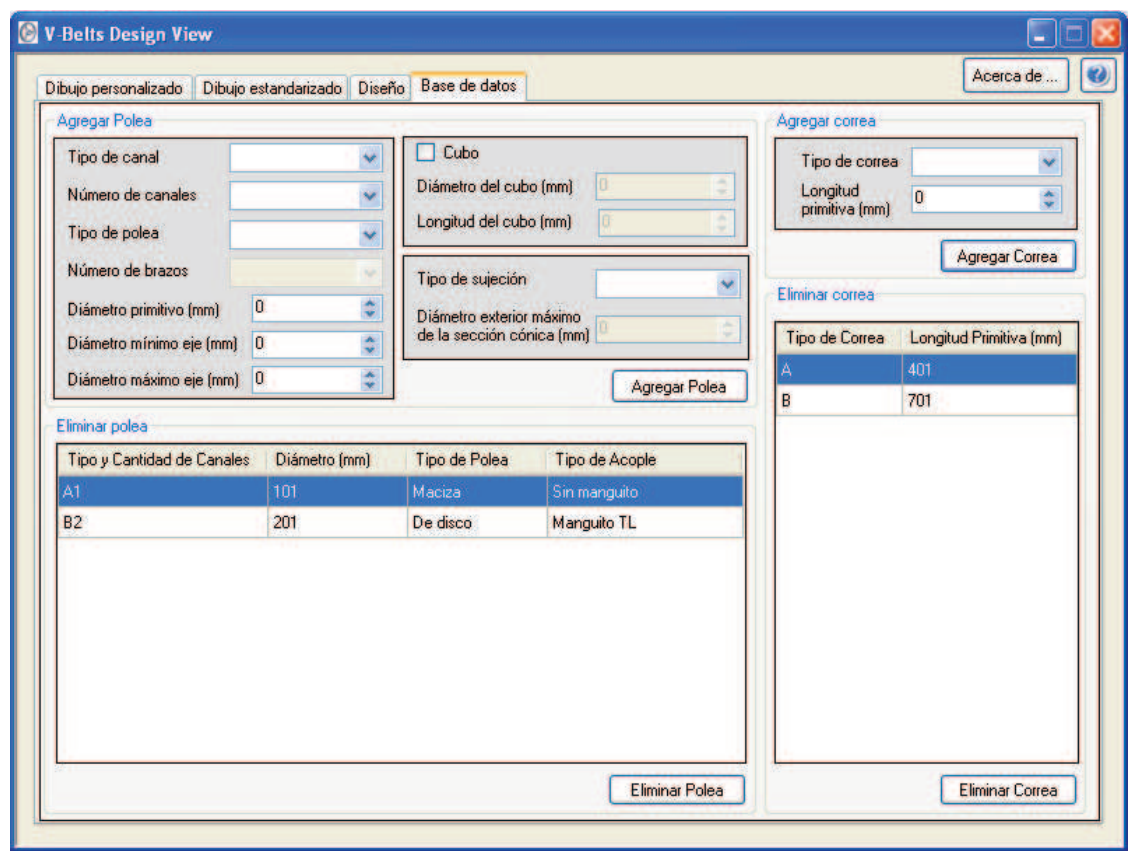

Fig. 5. Módulo de ingreso de elementos a la base de datos

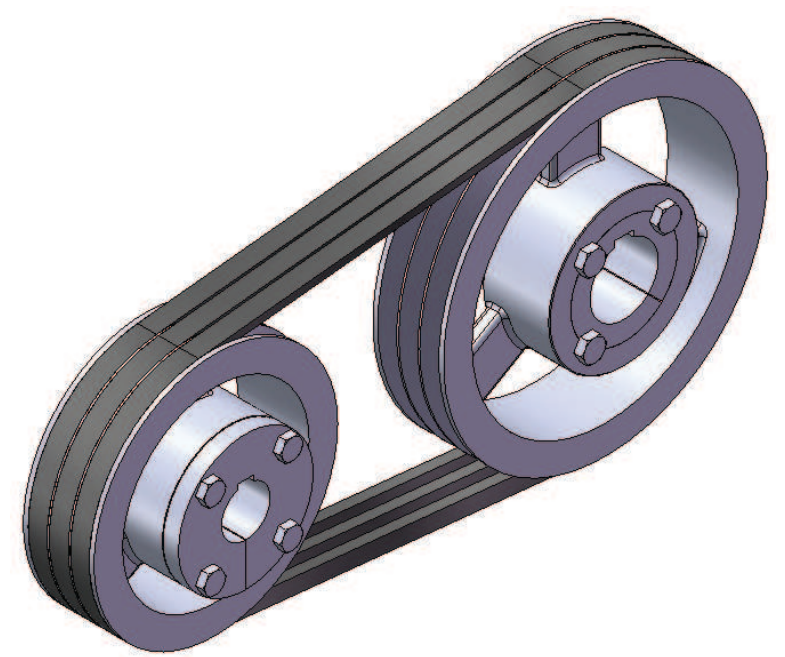

Fig. 6. Transmisión generada por el software desarrollado

Finalmente, la generación del modelo sólido de la transmisión se muestra como resultado de los primeros 3 módulos.

\section{AnÁlisis de RESUltados}

Debido a la enorme cantidad de cálculos realizados por el programa desarrollado, se considera ineficiente y tediosa la validación de estos de manera manual; por este motivo se usó una aplicación similar, desarrollada por la firma Optibelt (Optibelt CAP 6.0 Drive Calculation), la cual brinda de manera gratuita versiones demostrativas con pocas funcionalidades $\mathrm{y}$ número limitado de ejecuciones.

Se realizaron cuatro pruebas, en las cuales se abarcaron los rangos de velocidad y potencia 
admisible para transmisiones por correa en $\mathrm{V}$, variando el acople para cada prueba y los diferentes tipo de máquina motriz y accionada (Ver Tablas 5 y 6).

\section{TABla 5}

RESUlTAdOS DE LAS 2 PRIMERAS PRUEBAS SEGÚN OPTIBELT [1]

\begin{tabular}{|c|c|c|c|c|c|c|c|}
\hline Perfil & Longitud [mm] & $\mathbf{z}$ & $\mathrm{d} 1[\mathrm{~mm}]$ & $\mathrm{d} 2[\mathrm{~mm}]$ & $\mathrm{a}[\mathrm{mm}]$ & $\mathrm{n} 2[\mathrm{rpm}]$ & So'. [N] \\
\hline A & 980 & 4 & 71 & 160 & 305 & 1575 & 145 \\
\hline A & 995 & 3 & 75 & 170 & 301 & 1566 & 177 \\
\hline A & 1005 & 3 & 80 & 180 & 294 & 1578 & 175 \\
\hline Perfil & Longitud [mm] & $\mathbf{z}$ & $\mathrm{d} 1[\mathrm{~mm}]$ & $\mathrm{d} 2[\mathrm{~mm}]$ & $\mathrm{a}[\mathrm{mm}]$ & $\mathrm{n} 2[\mathrm{rpm}]$ & So' [N] \\
\hline B & 1665 & 8 & 170 & 250 & 501 & 1258 & 451 \\
\hline B & 1690 & 8 & 180 & 265 & 494 & 1257 & 445 \\
\hline B & 1740 & 6 & 190 & 280 & 499 & 1255 & 544 \\
\hline
\end{tabular}

\section{TABLA 6}

RESUlTADOS DE LAS 2 PRIMERAS PRUEBAS SEgúN V-Belts Design VIEW

\begin{tabular}{|c|c|c|c|c|c|c|c|}
\hline Perfil & Longitud [mm] & $\mathbf{d} 1$ [mm] & d2 [mm] & a [mm] & n2 [rpm] & So'. [N] \\
\hline A & 995 & 4 & 75 & 170 & 301 & 1566 & 102 \\
\hline A & 1005 & 3 & 80 & 180 & 294 & 1578 & 125 \\
\hline A & 1030 & 3 & 85 & 190 & 294 & 1588 & 123 \\
\hline \hline Perfil & Longitud [mm] & z & d1 [mm] & d2 [mm] & a [mm] & n2 [rpm] & So' [N] \\
\hline B & 1732 & 6 & 190 & 280 & 495 & 1255 & 424 \\
\hline B & 1840 & 5 & 212 & 315 & 503 & 1245 & 470 \\
\hline B & 1801 & 6 & 212 & 300 & 496 & 1307 & 404 \\
\hline
\end{tabular}

De acuerdo con los resultados, se observa que el software de la firma Optibelt proporciona transmisiones más pequeñas para las mismas condiciones de trabajo, lo cual indica que las constantes para el cálculo de la potencia nominal transmisible por correa admiten una potencia mayor que las constantes utilizadas en el software desarrollado. La alta tensión estática recomendada permite concluir que la firma establece transmisiones más pequeñas a expensas de un aumento en el tensado estático, lo cual reduce considerablemente la vida útil de las correas.

Los resultados establecen que el software desarrollado tiene mayor margen de seguridad que el programa brindado por Optibelt, al recomendar transmisiones con mayor cantidad de correas y dimensiones superiores sin incrementar.
La robustez de la transmisión, igualmente, indica un tiempo de vida útil mayor al utilizar tensiones estáticas de menor magnitud.

\section{Conclusiones}

El desarrollo de un software que permite realizar el cálculo para el diseño de transmisiones por correa en $\mathrm{V}$ y su representación gráfica tridimensional en el software SolidWorks, acompañado de una metodología de diseño planteada con base en una minuciosa revisión bibliográfica, le entrega al diseñador, ingeniero o estudiante de ingeniería una herramienta de cálculo con la cual determinar las configuraciones dimensionales $y$ las características de trabajo de las transmisiones por correa trapecial de acuerdo con las condiciones de operación ingresadas, reduciendo así el tiempo de diseño e incrementado la eficiencia del proceso, que puede llegar a ser tedioso y repetitivo.

A diferencia de otros softwares similares, desarrollados por fabricantes de transmisiones en Europa y Estados Unidos, el desarrollado en esta aplicación integra los cálculos de diseño de transmisiones con el dibujo en tercera dimensión de la solución seleccionada, mediante el acople de la aplicación con el software SolidWorks.

Se verificó la veracidad de los cálculos realizados por la aplicación desarrollada, confrontando los resultados obtenidos por el programa elaborado y por el software de cálculo de transmisiones desarrollado por la firma Optibelt (Optibelt CAP 6.0 Drive Calculation), consiguiendo con la aplicación creada en este proyecto transmisiones más confiables y duraderas, en comparación con los resultados arrojados por el software de Optibelt.

\section{REFERENCIAS}

[1] The Gates Rubber Company. Heavy Duty $V$-Belt Drive Design Manual. Denver, 
Colorado: Power Transmission Division. 1999.

[2] G. González Rey. Apuntes para el cálculo de transmisiones por correa en $V$. La Habana: Instituto Superior Politécnico José A. Echeverría (ISPJAE). 1999.

[3] British Standard. BS 3790: Specification for belt drives - Endless wedge belts, endless V-belts, banded wedge belts, banded V-belts and their corresponding pulleys. London, UK: 2006.

[4] Dobrovoslki. Elementos de máquinas. La Habana: MIR. 1976.

[5] The Goodyear Tire \& Rubber Company. Industrial V-Belts Engineering Manual [en línea]. USA. Disponible en: http://www. goodyearep.com/ptp_manual/ [consultado el 22 de septiembre de 2011].
[6] International Standard. ISO 5292: Belt drives - V-belts and V-ribbed belts Calculation of power ratings. 1995.

[7] Arntz Optibelt Gruppe. Optibelt CAP 6.0 Drive Calculation: Software para la selección de transmisiones por correa. Alemania. 2011. Disponible en: http:// www.optibelt.de/fileadmin/bilder/service/ software/CAP6/CAP6-0.exe. [consultado el 22 de septiembre de 2011].

[8] Arntz Optibelt Gruppe. Product range industrial Belts + Metal. Germany, 2005.

[9] B.J. Hamrock, B. Jacobson, S. R. Schmid. Elementos de Máquinas. México: McGraw Hill. 2000.

[10] O. E. Mayer. Teoría de las transmisiones flexibles por correa. Buenos Aires: Universidad de Buenos Aires. 2008. 